\title{
LIMITING THEOREMS FOR AGE-DEPENDENT BRANCHING PROCESSES ${ }^{1}$
}

\author{
BY \\ Norman Levinson
}

1. To motivate the theorems that will be stated and proved here, consider particles which are assumed to have a life span with cumulative probability distribution function $G(t)$. At the end of its life a particle is assumed to split into $n$ particles with probability $q_{n}$, where each particle has the same properties as the original. It is assumed $q_{n} \geqq 0$ and $n \geqq 0$. The generating function associated with $\left\{q_{n}\right\}$ is

$$
h(s)=\sum_{j=0}^{\infty} q_{j} s^{j}, \quad h(1)=1 .
$$

Given a particle at $t=0$, let the probability that there are $n$ particles at time $t \geqq 0$ be $p_{n}(t) \geqq 0$. The generating function is

$$
F(s, t)=\sum_{0}^{\infty} p_{j}(t) s^{j}, \quad F(1, t)=1 .
$$

Then the above description suggests that $F(s, t)$ satisfies

$$
F(s, t)=\int_{0-}^{t} h(F(s, t-y)) d G(y)+s[1-G(t)] .
$$

This problem with $h(s)=s^{2}$ and with mild restrictions on $G(t)$ has been studied by Bellman and Harris [1]. References to the literature will be found in [1].

In the special case where $G(t)$ is a step function with one discontinuity, the process becomes the Galton-Watson branching process. For this case the author has shown [2] that a best possible condition on $h(s)$ for the desired limiting theorems to hold is just a little more stringent than the existence of the first moment

$$
\mu=h^{\prime}(1)=\sum_{1}^{\infty} j q_{j}<\infty .
$$

It will be shown here that, with $\mu>1$, essentially the same condition on $h(s)$ as given in [2] is sufficient to yield the basic limit theorem in the agedependent case subject to restrictions on $G(t)$.

If, following [1], the random variable representing the number of particles at time $t$, starting with one particle at $t=0$, is denoted by $Z(t)$, then for $t \geqq 0$ and $|s| \leqq 1$

$$
\begin{gathered}
F(s, t)=E\left[s^{Z(t)}\right], \\
E[Z(t)]=m(t)=\partial F(1, t) / \partial s .
\end{gathered}
$$

Received September 2, 1958.

1 The preparation of this paper was supported by the Office of Naval Research. 
It will be shown that there is a limiting distribution of $Z(t) / m(t)$ as $t \rightarrow \infty$. In terms of $F$, what will be shown is that for $\mathrm{Rl} s \geqq 0$ there exists

$$
\lim _{t \rightarrow \infty} F\left(e^{-s / m(t)}, t\right)=\phi(s),
$$

so that $\phi$ is the Laplace Stieltjes transform of the limiting cumulative distribution function.

If $a$ is chosen so that

$$
\mu \int_{0}^{\infty} e^{-a y} d G(y)=1
$$

where $\mu$ is defined in (1.4), then it will be shown that

$$
\phi(s)=\int_{0}^{\infty} h\left(\phi\left(s e^{-a y}\right)\right) d G(y), \quad \phi(0)=1, \quad \phi^{\prime}(0)=-1 .
$$

For given $G$ and $h$, the main object of this paper is to prove (1) that (1.3) has a solution $F(s, t),(2)$ that (1.8) has a solution $\phi$, and (3) that (1.6) is valid, that is, that as $t \rightarrow \infty, Z(t) / m(t)$ has a limiting cumulative distribution with Laplace Stieltjes transform $\phi(s)$. These results will now be stated and proved.

2. It will be assumed that $G(0+)=0$ and that there exists a continuous $g(t) \geqq 0$ on $t \geqq 0$ such that

$$
G(t)=\int_{0}^{t} g(y) d y, \quad G(\infty)=1 .
$$

It will be assumed that

$$
\int_{0}^{\infty} e^{-2 a t} g^{2}(t) d t<\infty
$$

where $a$ is given by (1.7). The requirement (2.2) can be weakened considerably as is indicated in $[1, \S 3]$.

The equation (1.3) now becomes

$$
F(s, t)=\int_{0}^{t} h(F(s, t-y)) g(y) d y+s[1-G(t)] .
$$

Theorem 2.1. With (1.4) and (2.1) valid, the equation (2.3) has a solution $F(s, t)$, continuous in $(s, t)$ for $|s| \leqq 1,0 \leqq t<\infty .|F(s, t)| \leqq 1$, and, for each $t, F(s, t)$ is analytic for $|s|<1$, and

$$
\partial^{j} F(0, t) / \partial s^{j} \geqq 0, \quad j=0,1,2, \cdots .
$$

The solution $F(s, t)$ is unique, and $F(1, t)=1$. Hence for each $t, F(s, t)$ is a generating function.

Proof. Successive approximations will be used. Let $F_{0}(s, t)=0$, and let

$$
F_{n+1}(s, t)=\int_{0}^{t} h\left(F_{n}(s, t-y)\right) g(y) d y+s[1-G(t)] .
$$


By (1.1), for complex $s,|s| \leqq 1,|h(s)| \leqq 1$, and by (1.4)

$$
\left|h^{\prime}(s)\right| \leqq \mu .
$$

Hence $F_{1}(s, t)=h(0) G(t)+s[1-G(t)]$, and therefore for $|s| \leqq 1$

$$
\left|F_{1}(s, t)\right| \leqq G(t)+|s|[1-G(t)] \leqq 1 .
$$

From (2.5) follows so long as $\left|F_{n}(s, t)\right| \leqq 1$,

$$
\left|F_{n+1}(s, t)\right| \leqq h(1) G(t)+|s|[1-G(t)] \leqq 1 .
$$

Hence by induction, $\left|F_{n}(s, t)\right| \leqq 1$.

Since $F_{0}=0,(2.7)$ shows that

$$
\left|F_{1}(s, t)-F_{0}(s, t)\right| \leqq 1
$$

From (2.5) follows

$$
\begin{aligned}
\mid F_{n+1}(s, t)- & F_{n}(s, t) \mid \\
& \leqq \int_{0}^{t}\left|h\left(F_{n}(s, t-y)\right)-h\left(F_{n-1}(s, t-y)\right)\right| g(y) d y .
\end{aligned}
$$

If $t_{1}>0$ is chosen, and if $K_{1}$ is chosen so that $|g(t)| \leqq K_{1}$ for $0 \leqq t \leqq t_{1}$, then (2.9) gives for $t \leqq t_{1}$

$$
\left|F_{n+1}(s, t)-F_{n}(s, t)\right| \leqq \mu K_{1} \int_{0}^{t}\left|F_{n}(s, t-y)-F_{n-1}(s, t-y)\right| d y .
$$

If we use (2.8), it follows by induction that

$$
\left|F_{n+1}(s, t)-F_{n}(s, t)\right| \leqq\left(\mu^{n} K_{1}^{n} / n !\right) t^{n} .
$$

Thus for $|s| \leqq 1,0 \leqq t \leqq t_{1}, F_{n}(s, t)$ converges uniformly to a limit $F(s, t)$ which must be continuous since $F_{n}(s, t)$ is. By letting $n \rightarrow \infty$ in (2.5) it follows that $F(s, t)$ satisfies $(2.3)$.

Since, as is readily verified by induction, $\mid F_{n}(s, t \mid<1$ for $|s|<1$ and is analytic in $s$ for fixed $t$, it follows by the uniform convergence that $F(s, t)$ is analytic in $s$ for $|s|<1$ for each $t$. Moreover, as is readily verified by induction,

$$
\partial^{j} F_{n}(0, t) / \partial s^{j} \geqq 0, \quad j \geqq 0 .
$$

Using the Cauchy formula yields

$$
\frac{\partial^{j} F_{n}}{\partial s^{j}}(0, t)=\frac{j !}{2 \pi} \int_{0}^{2 \pi} F_{n}\left(e^{i \theta}, t\right) e^{-i n \theta} d \theta .
$$

Hence the uniform convergence of $F_{n}$ to $F$ shows that

$$
\partial^{j} F(0, t) / \partial s^{j} \geqq 0 .
$$

If for any $s_{1},\left|s_{1}\right| \leqq 1,(2.3)$ has two continuous solutions $F\left(s_{1}, t\right)$ and $\widetilde{F}\left(s_{1}, t\right)$, where $\left|F\left(s_{1}, t\right)\right| \leqq 1$ and $\left|\widetilde{F}\left(s_{1}, t\right)\right| \leqq 1$, then 
(2.11) $\left|F\left(s_{1}, t\right)-\widetilde{F}\left(s_{1}, t\right)\right| \leqq \mu \int_{0}^{t}\left|F\left(s_{1}, t-y\right)-\widetilde{F}\left(s_{1}, t-y\right)\right| g(y) d y$,

That $F-\widetilde{F}=0$ on $[0, \infty)$ will follow from Lemma 2.1 given below with $K=0$. Hence uniqueness is demonstrated.

Finally, since for $s=1$ a solution of (2.3) is obviously $F=1$, it follows from uniqueness that $F(1, t)=1$.

Lemma 2.1. Let $A(t)$ be real and continuous on $[0, \infty)$, let $B(t) \geqq 0$, and let

$$
\int_{0}^{\infty} B(t) d t<\infty
$$

Let $K \geqq 0$ be a constant. Let

$$
A(t) \leqq \int_{0}^{t} A(t-y) B(y) d y+K .
$$

Then there is a constant $k$ such that

$$
A(t) \leqq 2 K e^{k t} .
$$

Proof. Choose $k \geqq 0$ so that

$$
\int_{0}^{\infty} e^{-k y} B(y) d y<\frac{1}{2}
$$

Let $R(t)=A(t) e^{-k t}$. Then

$$
R(t) \leqq \int_{0}^{t} R(t-y) e^{-k y} B(y) d y+K .
$$

Let $t_{1}>0$, and let $\max R(t)$ on $\left[0, t_{1}\right]$ be denoted by $M$. Then clearly

$$
M \leqq M \int_{0}^{t_{1}} e^{-k y} B(y) d y+K \leqq \frac{1}{2} M+K .
$$

Hence $M \leqq 2 K$ on $\left[0, t_{1}\right]$ for any choice of $t_{1}$. Hence $\mathrm{R}(t) \leqq 2 K$ on $[0, \infty)$, which proves the lemma.

Theorem 2.2. With (1.4), (2.1), and (2.2) valid, there exists a continuous function $m(t), 0 \leqq t<\infty$, such that in $|s| \leqq 1$

$$
\partial F(1, t) / \partial s=m(t)
$$

and

$$
\lim _{\sigma \rightarrow 0+} \frac{1-F\left(e^{-\sigma}, t\right)}{\sigma}=m(t)
$$

uniformly on any finite interval $0 \leqq t \leqq t_{1}$. Moreover $m(t)$ satisfies

$$
m(t)=\mu \int_{0}^{t} m(t-y) g(y) d y+1-G(t),
$$


and if $a$ is defined by (1.7), then

$$
\lim _{t \rightarrow \infty} e^{-a t} m(t)=c,
$$

where the constant

$$
c=(\mu-1) /\left[a \mu^{2} \int_{0}^{\infty} y g(y) e^{-a y} d y\right],
$$

Proof. Formula (2.13) is an immediate consequence of (2.14) since $F(1, t)=1$ and hence will be proved once (2.14) is proved. By (2.4)

$$
F(s, t)=\sum_{0}^{\infty} p_{n}(t) s^{n},
$$

where $p_{n}(t) \geqq 0$. (By the Cauchy formula for the coefficients, the continuity of $F(s, t)$ implies that the $p_{n}(t)$ are continuous on $[0, \infty)$, but this will not be required here.) Since $F(1, t)=1$,

$$
\sum_{0}^{\infty} p_{n}(t)=1
$$

From (2.3) follows for $\sigma>0$

$$
\begin{aligned}
\frac{1-F\left(e^{-\sigma}, t\right)}{\sigma}=\int_{0}^{t} \frac{1-h\left(F\left(e^{-\sigma}, t-y\right)\right)}{\sigma} & g(y) d y \\
& +\frac{1-e^{-\sigma}}{\sigma}(1-G(t)) .
\end{aligned}
$$

Since

$$
\frac{1-h\left(F\left(e^{-\sigma}, t\right)\right)}{1-F\left(e^{-\sigma}, t\right)} \leqq \mu
$$

it follows from (2.20) that

$$
\frac{1-F\left(e^{-\sigma}, t\right)}{\sigma} \leqq \mu \int_{0}^{t} \frac{1-F\left(e^{-\sigma}, t-y\right)}{\sigma} g(y) d y+(1-G(t)) .
$$

Hence by Lemma 2.1 there exists $k>0$ such that

$$
\frac{1-F\left(e^{-\sigma}, t\right)}{\sigma} \leqq 2 e^{k t} ; \quad \sigma>0 .
$$

From (2.18)

$$
\frac{1-F\left(e^{-\sigma}, t\right)}{\sigma}=\sum_{0}^{\infty} n p_{n}(t) \frac{1-e^{-n \sigma}}{n \sigma} .
$$

From (2.21) and (2.22)

$$
\sum_{0}^{\infty} n p_{n}(t) \leqq 2 e^{k t}
$$$$
t \geqq 0
$$

Let

$$
m(t)=\sum_{0}^{\infty} n p_{n}(t)
$$


Then from (2.22)-(2.24) follows, since $1-e^{-n \sigma} \leqq n \sigma$,

$$
\frac{1-F\left(e^{-\sigma}, t\right)}{\sigma} \leqq m(t)
$$

and

$$
\lim _{\sigma \rightarrow 0+} \frac{1-F\left(e^{-\sigma}, t\right)}{\sigma}=m(t)
$$

From (2.20)

$$
\begin{aligned}
\frac{1-F\left(e^{-\sigma}, t\right)}{\sigma}=\int_{0}^{t} \frac{1-h\left(F\left(e^{-\sigma}, y\right)\right)}{\sigma} g(t & -y) d y \\
& +\frac{1-e^{-\sigma}}{\sigma}(1-G(t)) .
\end{aligned}
$$

Since

$$
\frac{1-h\left(F\left(e^{-\sigma}, y\right)\right)}{\sigma}=\frac{1-h(F)}{1-F} \frac{1-F}{\sigma} \leqq \mu m(y) \leqq 2 \mu e^{k y},
$$

it follows from (2.27) that since $g$ is continuous over any interval $\left[0, t_{1}\right]$,

$$
\frac{1-F\left(e^{-\sigma}, t\right)}{\sigma}
$$

is continuous in $t$ over $\left[0, t_{1}\right]$ and that this continuity is uniform in $\sigma$, $0<\sigma \leqq \sigma_{1}$, as well as in $t, 0 \leqq t \leqq t_{1}$. This uniform continuity implies that the convergence in (2.26) must be uniform in $t$ over any finite interval of $t$, and that $m(t)$ must be continuous. Writing

$$
\frac{1-h}{\sigma}=\frac{1-h}{1-F} \frac{1-F}{\sigma}
$$

in (2.20) and letting $\sigma \rightarrow 0+$ yields (2.15) as a result of the uniform convergence of $(2.26)$.

It remains now to use (2.15) to obtain (2.16) and (2.17). This well known renewal equation is treated by the Laplace transform. Here it is already known that $m(t)$ satisfies (2.15), and hence it is not necessary to establish the existence of a solution. Moreover Lemma 2.1 shows the uniqueness of $m(t)$ as a solution of $(2.15)$.

[Addendum to $\$ 2$, October 30,1958 . The referee has remarked that it would be desirable to show that $m(t)$ is an increasing function since the probabilistic origin of the problem indicates this to be the case. To show this analytically, one takes the derivative of (2.15) formally and finds that $m^{\prime}$, if it exists, is a solution of

$$
\text { (*) } \quad \xi(t)=\int_{0}^{t} \xi(y) g(t-y) d y+(\mu-1) g(t) .
$$


(Here use is made of $m(0)=1$.) But since $g \geqq 0$, the usual Volterra procedure shows that $(*)$ has a solution $\xi \geqq 0$ which is unique. Setting $y=t-x$ in $(*)$ and integrating with respect to $t$ gives, if one defines

$$
\begin{gathered}
\tilde{m}(t)=1+\int_{0}^{t} \xi(y) d y, \\
\widetilde{m}(t)=1+\mu \int_{0}^{t} d y \int_{0}^{y} \xi(y-x) g(x) d x+(\mu-1) G(t) \\
=1+\mu \int_{0}^{t} g(x)\left(\int_{x}^{t} \xi(y-x) d y\right) d x+(\mu-1) G(t) \\
=1+\mu \int_{0}^{t} g(x)[\tilde{m}(t-x)-1] d x+(\mu-1) G(t),
\end{gathered}
$$

or

$$
\widetilde{m}(t)=\mu \int_{0}^{t} \widetilde{m}(t-y) g(y) d y+1-G(t) .
$$

Hence, $\tilde{m}(t)=m(t)$, and thus $m^{\prime}(t)=\xi(t) \geqq 0$.]

With $a$ defined by (1.7), let

$$
f(t)=e^{-a t} m(t) .
$$

Then by (2.15)

$$
f(t)=\mu \int_{0}^{t} f(t-y) e^{-a y} g(y) d y+e^{-a t}[1-G(t)] .
$$

From (2.23), (2.24), and (2.28) it follows that the Laplace transform

$$
\Phi(w)=\int_{0}^{\infty} f(t) e^{-t w} d t
$$

exists. Let $w=u+i v$, and let

Then (2.29) yields

$$
\Gamma(w)=\int_{0}^{\infty} g(t) e^{-t w} d t .
$$

$$
\Phi(w)=\mu \Gamma(a+w) \Phi(w)+\frac{1-\Gamma(a+w)}{a+w} .
$$

Since $\Gamma(w)$ is analytic for $u>0$, the above shows that

$$
\Phi(w)=\frac{1-\Gamma(a+w)}{[1-\mu \Gamma(a+w)](a+w)}
$$

is analytic for $u>0$. Since $g(t) \geqq 0$, it follows easily that

and by (1.7)

$$
1-\mu \Gamma(a+i v) \neq 0, \quad v \neq 0,
$$

$$
\mu|\Gamma(a+u+i v)|<1, \quad u>0 .
$$


Hence $\Phi(w)$ is analytic for $u \geqq 0$ except for a pole at $w=0$. The residue of this pole is

$$
\frac{1-\Gamma(a)}{-\mu a \Gamma^{\prime}(a)}=c
$$

where $c$ is given by (2.17). Hence

$$
\Phi(w)-c / w
$$

is analytic for $u \geqq 0$. By the Riemann-Lebesgue theorem

Clearly

$$
\lim _{v \rightarrow \infty} \frac{1-\Gamma(a+i v)}{1-\mu \Gamma(a+i v)}=1 \text {. }
$$

$$
\Phi(w)-\frac{c}{w}-\frac{1-c}{a+w}=\frac{(\mu-1) \Gamma(a+w)}{(a+w)[1-\mu \Gamma(a+w)]}-\frac{a c}{w(a+w)} .
$$

The right side is analytic on $u=0$, and for large $v, \Gamma(a+i v)$ is $L^{2}(-\infty, \infty)$ by (2.2). Hence by the Schwarz inequality the right side is absolutely integrable on $u=0$. Thus its Fourier transform

$$
f(t)-c-(1-c) e^{-a t} \rightarrow 0
$$

as $t \rightarrow \infty$. Thus (2.16) and (2.17) are proved.

3. Let $h(s)$ be defined by (1.1) for $|s| \leqq 1$. Let $\beta(t)$ be a continuous, positive, monotone decreasing function such that

$$
\int_{1}^{\infty} \frac{\beta(t)}{t} d t \leqq \infty
$$

and let

$$
\sum_{j \geqq n}^{\infty} j q_{j} \leqq \beta(n) .
$$

(Examples of $\beta(t)$ are $C t^{-\delta}, C[\log (1+t)]^{-1-\delta}$, etc., where $C$ and $\delta>0$ are constants. Clearly (3.1) and (3.2) are considerably weaker than the requirement of the existence of the second moment which would imply (3.2) with $\beta(n)=C / n$.) It will also be convenient to assume that $\beta$ is of class $C^{\prime \prime}$ and that

$$
3 \beta^{\prime}(t)+t \beta^{\prime \prime}(t) \leqq 0
$$

for large $t$.

In [2, Lemma 2.1] it is shown that (3.1) and (3.2) imply the existence of a continuous nondecreasing function $\alpha(\sigma), 0 \leqq \sigma<\infty, \alpha(0)=0$, such that

$$
\begin{aligned}
\int_{0}^{1} \frac{\alpha(\sigma)}{\sigma} d \sigma<\infty, & \\
\left|\mu-\frac{1-h\left(e^{-\sigma}\right)}{\sigma}\right| \leqq \alpha(\sigma), & 0 \leqq \sigma<\infty .
\end{aligned}
$$


The condition (3.3) implies that $\alpha$ is $C^{\prime \prime}$ and that for small $\sigma$

$$
\alpha^{\prime \prime}(\sigma) \leqq 0 .
$$

In the course of proving (1.6) it will be convenient to consider

$$
B(s, t)=F\left(\exp \left(-s e^{-a t} / c\right), t\right) .
$$

(Since $m(t) e^{-a t} \rightarrow c$, it will be easy to relate the above to (1.6).) From (2.14) follows

$$
\lim _{\sigma \rightarrow 0+} \frac{1-B(\sigma, t)}{\sigma}=\lim _{\sigma \rightarrow 0+} \frac{1-F\left(\exp \left(-\sigma e^{-a t} / c\right), t\right)}{\sigma}=\frac{m(t) e^{-a t}}{c}=\frac{f(t)}{c},
$$

where $f(t)$ is defined in (2.28). Moreover this convergence is uniform over any finite interval of $t$. It will be convenient to set

$$
\pi(t)=f(t) / c
$$

so that

$$
\lim _{\sigma \rightarrow 0+} \frac{1-B(\sigma, t)}{\sigma}=\pi(t) .
$$

As already remarked, the convergence in (3.9) is uniform over any finite interval of $t$. An important step of the proof of (1.6) is the following theorem.

TheOREM 3.1. The convergence in (3.9) is uniform in $t$ for $0 \leqq t<\infty$; that is, for given $\varepsilon>0$ there exists $\delta>0$ such that

$$
\left|\pi(t)-\frac{1-B(\sigma, t)}{\sigma}\right|<\varepsilon
$$

for $0<\sigma<\delta$ independent of $t$.

Proof of Theorem 3.1. From (2.3) and (3.7) follows for Rl $s \geqq 0$

$$
B(s, t)=\int_{0}^{t} h\left(B\left(s e^{-a y}, t-y\right)\right) g(y) d y+\exp \left(-s e^{-a t} / c\right)[1-G(t)],
$$

and from (3.10)

$$
\begin{aligned}
\frac{1-B(s, t)}{s}=\int_{0}^{t} \frac{1-h\left(B\left(s e^{-a y}, t-y\right)\right.}{s} g(y) d y & \\
& +\frac{1-\exp \left(-s e^{-a t} / c\right)}{s}[1-G(t)]
\end{aligned}
$$

Let

$$
Q(\sigma, t)=\pi(t)-\frac{1-B(\sigma, t)}{\sigma} .
$$

Then $Q(\sigma, t)$ is continuous in $(\sigma, t)$ for $\sigma \geqq 0, t \geqq 0$, and by (3.9)

$$
Q(0, t)=0 \text {. }
$$


By (2.29)

$$
\pi(t)=\mu \int_{0}^{t} \pi(t-y) e^{-a y} g(y) d y+\frac{e^{-a t}}{c}[1-G(t)] .
$$

Hence by (3.11) and (3.13)

$$
\begin{aligned}
& Q(\sigma, t)=\mu \int_{0}^{t} Q\left(\sigma e^{-a y}, t-y\right) e^{-a y} g(y) d y \\
& +\int_{0}^{t}\left[\mu \frac{1-B\left(\sigma e^{-a y}, t-y\right)}{\sigma e^{-a y}}-\frac{1-h\left(B\left(\sigma e^{-a y}, t-y\right)\right)}{\sigma e^{-a y}}\right] e^{-a y} g(y) d y \\
& +\left[\frac{e^{-a t}}{c}-\frac{1-\exp \left(-\sigma e^{-a t} / c\right)}{\sigma}\right][1-G(t)] .
\end{aligned}
$$

From (2.18) and (3.7)

$$
B(s, t)=\sum_{0}^{\infty} p_{n}(t) \exp \left(-n s e^{-a t} / c\right) .
$$

From (3.15) and $p_{n}(t) \geqq 0,(3.9)$ implies

$$
\pi(t)=(1 / c) \sum_{0}^{\infty} n p_{n}(t) e^{-a t}
$$

and

$$
Q(\sigma, t)=\sum_{0}^{\infty} \frac{n e^{-a t}}{c} p_{n}(t)\left[1-\frac{1-\exp \left(-n \sigma e^{-a t} / c\right)}{n \sigma e^{-a t} / c}\right]
$$

The formula (3.17) shows that $Q(\sigma, t)$ is an increasing function of $\sigma$ since $\left(1-e^{-x}\right) / x$ is decreasing in $x$. Let

$$
\max _{0} \leqq t \leqq t_{0} Q(\sigma, t)=M\left(\sigma, t_{0}\right) .
$$

Then $M$ is continuous since $Q$ is. Since $Q$ is increasing in $\sigma, M\left(\sigma, t_{0}\right)$ is an increasing function of $\sigma$.

From (3.5) it is readily verified, since $1-x \leqq \log (1 / x)$, that

$$
0<\mu-\frac{1-h(x)}{1-x} \leqq \alpha\left(\log \frac{1}{x}\right)
$$

and hence

$$
\left|\mu-\frac{1-h(x)}{1-x}\right| \leqq \alpha[2(1-x)], \quad \frac{1}{2} \leqq x<1 .
$$

Note that since $1-x<e^{-x}<1-x+x^{2} / 2$,

$$
0<\frac{e^{-a t}}{c}-\frac{1-\exp \left(-\sigma e^{-a t} / c\right)}{\sigma} \leqq \frac{\sigma}{2 c^{2}} \text {. }
$$

Let $\tau$ be defined by

$$
\mu \int_{0}^{\tau} e^{-a y} g(y) d y=\frac{1}{2} .
$$

Choose $t_{0}>\tau$, and choose $t$ so that $Q(\sigma, t)=M\left(\sigma, t_{0}\right)$ in (3.17). If we de- 
note $M\left(\sigma, t_{0}\right)$ by $M_{0}(\sigma)$, (3.14) yields, on dividing the range of integration in the first integral into $(0, \tau)$ and $(\tau, t)$ (or simply taking $(0, t)$ if $t<\tau)$, $\begin{aligned} M_{0}(\sigma) & \leqq \frac{1}{2} M_{0}(\sigma)+\frac{1}{2} M_{0}\left(\sigma e^{-a \tau}\right)+\sigma / 2 c^{2} \\ & +\int_{0}^{t}\left[\mu-\frac{1-h\left(B\left(\sigma e^{-a y}, t-y\right)\right)}{1-B}\right] \frac{1-B\left(\sigma e^{-a y}, t-y\right)}{\sigma e^{-a y}} e^{-a y} g(y) d y .\end{aligned}$

Since by (3.17) $Q \geqq 0,1-B(\sigma, t) \leqq \pi(t) \sigma$. Hence

$$
\frac{1-B\left(\sigma e^{-a y}, t\right)}{\sigma e^{-a y}} \leqq \pi(t)<C_{0},
$$

where $C_{0}$ is a constant. Hence by using (3.19) and taking $\sigma$ small,

$$
M_{0}(\sigma) \leqq M_{0}\left(\sigma e^{-a r}\right)+\frac{\sigma}{c^{2}}+4 C_{0} \int_{0}^{t} \alpha\left(2 C_{0} \sigma e^{-a y}\right) e^{-a y} g(y) d y,
$$

or

$$
M_{0}(\sigma) \leqq M_{0}\left(\sigma e^{-a \tau}\right)+\sigma / c^{2}+4 C_{0} \alpha\left(2 C_{0} \sigma\right) .
$$

The above with $\sigma$ replaced by $\sigma e^{-a \tau}, \sigma e^{-2 a \tau}$, etc. yields

$$
\begin{aligned}
& M_{0}\left(\sigma e^{-a \tau}\right) \leqq M_{0}\left(\sigma e^{-2 a \tau}\right)+\sigma e^{-a \tau} / c^{2}+4 C_{0} \alpha\left(2 C_{0} \sigma e^{-a \tau}\right), \\
& M_{0}\left(\sigma e^{-2 a \tau}\right) \leqq M_{0}\left(\sigma e^{-3 a \tau}\right)+\sigma e^{-2 a \tau} / c^{2}+4 C_{0} \alpha\left(2 C_{0} \sigma e^{-2 a \tau}\right),
\end{aligned}
$$

etc. Since $M_{0}(0)=0$, adding gives

$$
M_{0}(\sigma) \leqq \frac{\sigma}{c^{2}} \frac{1}{1-e^{-a \tau}}+4 C_{0} \sum_{j=0}^{\infty} \alpha\left(2 C_{0} \sigma e^{-j a \tau}\right) .
$$

Denote $\sup _{0 \leqq t<\infty} Q(\sigma, t)$ by $M(\sigma)$. Then since the right side above does not depend on $t_{0}$,

$$
M(\sigma) \leqq \frac{\sigma}{c^{2}} \frac{1}{1-e^{-a \tau}}+4 C_{0} \sum_{j=0}^{\infty} \alpha\left(2 C_{0} \sigma e^{-j a \tau}\right) .
$$

(Since $\partial B / \partial \sigma<0$, it is easily seen that $\partial Q / \partial \sigma<1 / \sigma^{2}$ for $\sigma>0$, and hence $M(\sigma)$ is continuous for $\sigma>0$, but this is not required here.) Noting that for $j-1 \leqq u \leqq j$

we obtain

$$
\alpha\left(2 C_{0} \sigma e^{-j a r}\right) \leqq \alpha\left(2 C_{0} \sigma e^{-u a r}\right),
$$

$$
\sum_{j=1}^{\infty} \alpha\left(2 C_{0} \sigma e^{-j a \tau}\right) \leqq \int_{0}^{\infty} \alpha\left(2 C_{0} \sigma e^{-u a \tau}\right) d u \leqq \frac{1}{a \tau} \int_{0}^{2 C_{0} \sigma} \alpha(y) \frac{d y}{y} .
$$

Hence (3.21) shows that $M(0+)=0$. This proves Theorem 3.1.

4. In proving (1.6) it will first be shown that

$$
\lim _{t \rightarrow \infty} B(s, t)=\phi(s) .
$$

In order to prove (4.1), it will be convenient first to define $\phi(s)$ independent 
of any limiting process. Letting $t \rightarrow \infty$ in (3.10) suggests that if (4.1) is true, then

$$
\phi(s)=\int_{0}^{\infty} h\left(\phi\left(s e^{-a y}\right)\right) g(y) d y .
$$

Moreover $B(0, t)=1$ suggests that $\phi(0)=1$, and since $\pi(t)=f(t) / c \rightarrow 1$ as $t \rightarrow \infty,(3.9)$ suggests

$$
\phi^{\prime}(0)=-1 \text {. }
$$

Theorem 4.1. With $h(s)$ subject to (3.1), (3.2), and (3.3), the equation

$$
\phi(\sigma)=\int_{0}^{\infty} h\left(\phi\left(\sigma e^{-a y}\right)\right) g(y) d y
$$

has a continuous solution for $0 \leqq \sigma<\infty$ with $|\phi(\sigma)| \leqq 1, \phi(0)=1$, and $\phi^{\prime}(0)=-1$. Moreover this solution is unique.

Proof. Let $\phi_{0}(\sigma)=e^{-\sigma}$, and let

$$
\phi_{n+1}(\sigma)=\int_{0}^{\infty} h\left(\phi_{n}\left(\sigma e^{-a y}\right)\right) g(y) d y
$$

for $n \geqq 0$. By induction, $0 \leqq \phi_{n}(\sigma) \leqq 1$. Clearly

$$
\phi_{1}(\sigma)=\int_{0}^{\infty} h\left(\exp \left(-\sigma e^{-a y}\right)\right) g(y) d y
$$

and

$$
\frac{1-\phi_{1}(\sigma)}{\sigma}=\int_{0}^{\infty} \frac{1-h\left(\exp \left(-\sigma e^{-a y}\right)\right)}{\sigma e^{-a y}} e^{-a y} g(y) d y .
$$

By using (3.5)

$$
\left|\frac{1-\phi_{1}(\sigma)}{\sigma}-1\right| \leqq \int_{0}^{\infty} \alpha\left(\sigma e^{-a y}\right) e^{-a y} g(y) d y .
$$

Hence since $\alpha(\sigma)$ is nondecreasing, using (1.7) shows

$$
\left|\left(1-\phi_{1}(\sigma)\right) / \sigma-1\right| \leqq \alpha(\sigma) \text {. }
$$

It is readily verified that

Hence

$$
\left|\left(1-\phi_{0}(\sigma)\right) / \sigma-1\right| \leqq \frac{1}{2} \sigma .
$$

$$
\left|\phi_{1}(\sigma)-\phi_{0}(\sigma)\right| \leqq \sigma\left[\alpha(\sigma)+\frac{1}{2} \sigma\right] .
$$

If we denote $\alpha(\sigma)+\frac{1}{2} \sigma$ by $\alpha_{1}(\sigma)$, it is clear that $\alpha_{1}$ has the required properties of $\alpha$, namely (3.4) and (3.6). Hence

$$
\left|\phi_{1}(\sigma)-\phi_{0}(\sigma)\right| \leqq \sigma \alpha_{1}(\sigma) .
$$

By (4.5)

$$
\phi_{2}(\sigma)-\phi_{1}(\sigma)=\int_{0}^{\infty}\left[h\left(\phi_{1}\left(\sigma e^{-a y}\right)\right)-h\left(\phi_{0}\left(\sigma e^{-a y}\right)\right)\right] g(y) d y .
$$


Since $h^{\prime} \leqq \mu$, this and (4.7) give

$$
\left|\phi_{2}(\sigma)-\phi_{1}(\sigma)\right| \leqq \mu \int_{0}^{\infty} \sigma e^{-a y} \alpha_{1}\left(\sigma e^{-a y}\right) g(y) d y .
$$

With $\tau$ defined as below (3.20), this gives

$$
\left|\phi_{2}(\sigma)-\phi_{1}(\sigma)\right| \leqq(\sigma / 2) \alpha_{1}(\sigma)+(\sigma / 2) \alpha_{1}\left(\sigma e^{-a \tau}\right) .
$$

Since $\alpha_{1}^{\prime \prime} \leqq 0$,

$$
\left|\phi_{2}(\sigma)-\phi_{1}(\sigma)\right| \leqq \sigma \alpha_{1}\left(\sigma\left(1+e^{-a \tau}\right) / 2\right) .
$$

An easy induction now gives

$$
\left|\phi_{n+1}(\sigma)-\phi_{n}(\sigma)\right| \leqq \sigma \alpha_{1}\left[\sigma\left(\left(1+e^{-a \tau}\right) / 2\right)^{n}\right] .
$$

Hence denoting $\left(1+e^{-a r}\right) / 2$ by $e^{-\delta}$, we have

$$
\begin{aligned}
\sum_{j=n+1}^{\infty}\left|\phi_{j+1}(\sigma)-\phi_{j}(\sigma)\right| & \leqq \sigma \sum_{j=n+1}^{\infty} \alpha_{1}\left[\sigma e^{-\delta j}\right] \\
& \leqq \sigma \int_{n}^{\infty} \alpha_{1}\left(\sigma e^{-\delta t}\right) d t=\frac{\sigma}{\delta} \int_{0}^{\sigma e^{-\delta n}} \alpha_{1}(u) \frac{d u}{u}
\end{aligned}
$$

if $u=\sigma e^{-\delta t}$. Hence over any finite interval of $\sigma, 0 \leqq \sigma \leqq \sigma_{1}, \phi_{n}(\sigma)$ converges uniformly. Since each $\phi_{n}(\sigma)$ is continuous by induction, it follows that the limit $\phi(\sigma)$ is also continuous. By (4.5) it is clear that $\phi(\sigma)$ satisfies (4.4). By induction it is clear that $\phi_{n}(0)=1$, and hence $\phi(0)=1$.

From (4.10) with $n=0$

$$
\left|\frac{1-\phi(\sigma)}{\sigma}-\frac{1-\phi_{0}(\sigma)}{\sigma}\right| \leqq \frac{1}{\delta} \int_{0}^{\sigma} \alpha_{1}(u) \frac{d u}{u}+\alpha_{1}(\sigma) .
$$

Letting $\sigma \rightarrow 0+$ and recalling that $\phi_{0}(\sigma)=e^{-\sigma}$ gives $\phi^{\prime}(0)=-1$.

Now assume that (4.4) has two solutions $\phi$ and $\phi^{*}$ satisfying the conditions of the theorem. Then

Hence

$$
\phi(\sigma)-\phi^{*}(\sigma)=\int_{0}^{\infty}\left[h\left(\phi\left(\sigma e^{-a y}\right)\right)-h\left(\phi^{*}\left(\sigma e^{-a y}\right)\right)\right] g(y) d y .
$$

$$
\left|\frac{\phi(\sigma)-\phi^{*}(\sigma)}{\sigma}\right| \leqq \mu \int_{0}^{\infty}\left|\frac{\phi\left(\sigma e^{-a y}\right)-\phi^{*}\left(\sigma e^{-a y}\right)}{\sigma e^{-a y}}\right| e^{-a y} g(y) d y .
$$

Let

$$
\max _{0 \leqq u \leqq \sigma}\left|\frac{\phi(u)-\phi^{*}(u)}{u}\right|=N(\sigma) \text {. }
$$

Then since $\phi(0)=\phi^{*}(0)=1$ and since $\phi^{\prime}(0)=\phi^{* \prime}(0)=-1, N(0+)=0$. Also from (4.11) simple considerations including the introduction of $\tau$ lead to

Hence

$$
N(\sigma) \leqq \frac{1}{2} N(\sigma)+\frac{1}{2} N\left(\sigma e^{-a \tau}\right) .
$$

$$
N(\sigma) \leqq N\left(\sigma e^{-a \tau}\right) .
$$


Since $N(0+)=0$, iterations of the above lead to $N(\sigma) \equiv 0$, which proves the uniqueness of $\phi(\sigma)$ and completes the proof of Theorem 4.1.

It will now be shown that

$$
\lim _{t \rightarrow \infty} B(\sigma, t)=\phi(\sigma), \quad \sigma \geqq 0 .
$$

This is a consequence of the following theorem since $B(0, t)=\phi(0)=1$.

Theorem 4.2. For $0<\sigma<\infty$

$$
\lim _{t \rightarrow \infty}(B(\sigma, t)-\phi(\sigma)) / \sigma=0 .
$$

Proof. From the definition of $Q$

$$
(B(\sigma, t)-\phi(\sigma)) / \sigma=Q(\sigma, t)+[(1-\phi(\sigma)) / \sigma-1]+[1-\pi(t)] .
$$

Let

Let

$$
\rho(\sigma, t)=|B(\sigma, t)-\phi(\sigma)| / \sigma .
$$

$$
R(\sigma)=\lim \sup _{t \rightarrow \infty} \rho(\sigma, t) .
$$

Theorem 3.1, $\phi^{\prime}(0)=-1$, and $\pi(\infty)=1$ used in (4.13) imply

$$
R(0+)=0 .
$$

Since $0<\partial Q / \partial \sigma<1 / \sigma^{2}$, it follows readily that $R(\sigma)$ is continuous for $\sigma>0$ and hence by (4.14) for $\sigma \geqq 0$.

Using (3.10) and (4.4) yields

$$
(B(\sigma, t)-\phi(\sigma)) / \sigma=J_{1}+J_{2},
$$

where

Clearly

$$
\begin{aligned}
& J_{1}=\int_{0}^{t}\left[h\left(B\left(\sigma e^{-a y}, t-y\right)\right)-h\left(\phi\left(\sigma e^{-a y}\right)\right)\right] g(y) \frac{d y}{\sigma} \\
& J_{2}=-\int_{t}^{\infty} h\left(\phi\left(\sigma e^{-a y}\right)\right) g(y) \frac{d y}{\sigma}+\frac{\exp \left(-\sigma e^{-a t} / c\right)}{\sigma}[1-G(t)] .
\end{aligned}
$$

$$
\begin{aligned}
& J_{1}=\int_{0}^{t} \frac{h\left(B\left(\sigma e^{-a y}, t-y\right)\right)-h\left(\phi\left(\sigma e^{-a y}\right)\right)}{B\left(\sigma e^{-a y}, t-y\right)-\phi\left(\sigma e^{-a y}\right)} \\
& \cdot \frac{B\left(\sigma e^{-a y}, t-y\right)-\phi\left(\sigma e^{-a y}\right)}{\sigma e^{-a y}} e^{-a y} g(y) d y .
\end{aligned}
$$

Hence

$$
\left|J_{1}\right| \leqq \mu \int_{0}^{t} \rho\left(\sigma e^{-a y}, t-y\right) e^{-a y} g(y) d y
$$

Also

$$
\begin{aligned}
J_{2}=\int_{t}^{\infty} \frac{1-h\left(\phi\left(\sigma e^{-a y}\right)\right)}{\sigma e^{-a y}} & e^{-a y} g(y) d y \\
& -\frac{1-\exp \left(-\sigma e^{-a t} / c\right)}{\sigma e^{-a t}}[1-G(t)] e^{-a t} .
\end{aligned}
$$


Hence

$$
\left|J_{2}\right| \leqq \mu \int_{t}^{\infty} \frac{1-\phi\left(\sigma e^{-a y}\right)}{\sigma e^{-a y}} e^{-a y} g(y) d y+e^{-a t} \int_{t}^{\infty} g(y) d y / c .
$$

Thus (4.15) gives

$$
\begin{aligned}
\rho(\sigma, t) \leqq \mu \int_{0}^{t} \rho\left(\sigma e^{-a y}, t-y\right) e^{-a y} g(y) d y & \\
& +C_{1} \int_{t}^{\infty} e^{-a y} g(y) d y+e^{-a t} \int_{t}^{\infty} g(y) d y / c
\end{aligned}
$$

for some constant $C_{1}$ since $(1-\phi(\sigma)) / \sigma$ is uniformly bounded.

By recalling $R(\sigma)$ above (4.14) and its continuity for $0 \leqq \sigma<\infty$, it follows that if $R(\sigma) \not \equiv 0$, then there exists some $\delta>0$ such that for some $\sigma>0$, $R(\sigma)=\delta$. Let $\sigma_{1}$ be the least $\sigma$ such that $R\left(\sigma_{1}\right)=\delta$. Hence $R(\sigma)<\delta$ for $0 \leqq \sigma<\sigma_{1}$. In particular if $\tau$ is defined as below (3.20) there exists $\eta<\delta$ such that

$$
R(\sigma) \leqq \eta, \quad 0 \leqq \sigma \leqq \sigma_{1} e^{-a \tau} .
$$

From Theorem 3.1 and $\phi^{\prime}(0)=-1$, it follows readily that there exists $K_{0}$ such that

From (4.16) for large $t$

$$
\rho(\sigma, t) \leqq K_{0}
$$

$$
\begin{aligned}
\rho\left(\sigma_{1}, t\right) \leqq & \mu \int_{0}^{\tau} \rho\left(\sigma_{1} e^{-a y}, t-y\right) e^{-a y} g(y) d y+\mu \int_{\tau}^{t / 2} \rho\left(\sigma_{1} e^{-a y}, t-y\right) e^{-a y} g(y) d y \\
& +\mu K_{0} \int_{t / 2}^{t} e^{-a y} g(y) d y+C_{1} \int_{t}^{\infty} e^{-a y} g(y) d y+e^{-a t} \int_{t}^{\infty} g(y) d y / c .
\end{aligned}
$$

If we let $t \rightarrow \infty$, this gives by (4.17)

$$
\delta \leqq \frac{1}{2} \delta+\frac{1}{2} \eta
$$

or $\delta \leqq \eta$, which is impossible. Hence $R(\sigma) \equiv 0$, and the theorem is proved.

5. It remains now to prove (1.6) and to extend some of the results from real $\sigma$ to complex $s$. Both are rather simple to do at this point.

It was proved in Theorem 2.1 that $F(s, t)$ is a generating function so that

$$
F(s, t)=\sum_{0}^{\infty} p_{n}(t) s^{n},
$$$$
|s| \leqq 1
$$

and $F(1, t)=1$ for $0 \leqq t<\infty$. Hence

$$
B_{1}(s, t)=F\left(e^{-s / m(t)}, t\right)=\sum_{0}^{\infty} p_{n}(t) e^{-n s / m(t)},
$$

and

$$
B(s, t)=F\left[\exp \left(-s e^{-a t} / c, t\right]=\sum_{0}^{\infty} p_{n}(t) \exp \left(-n s e^{-a t} / c\right)\right.
$$


Thus $B_{1}(s, t)$ is the Laplace Stieltjes transform of

$$
H_{1}(u, t)=\sum_{n \leqq u m(t)} p_{n}(t)
$$

and $B(s, t)$ of

$$
H(u, t)=\sum_{n \leqq u c \exp (a t)} p_{n}(t) ;
$$

that is, for $\mathrm{Rl} s \geqq 0$,

$$
B(s, t)=\int_{0_{-}}^{\infty} e^{-s u} d H(u, t),
$$

where $t$ is held fixed in $H$, and

$$
B_{1}(s, t)=\int_{0_{-}}^{\infty} e^{-s u} d H_{1}(u, t) .
$$

Since $B(\sigma, t) \rightarrow \phi(\sigma)$ as $t \rightarrow \infty$, the fact that $H(u, t)$ is a cumulative distribution function for each $t$ implies that $\phi(\sigma)$ is the Laplace Stieltjes transform of a cumulative distribution function $\Psi(u)$, and that at points of continuity of $\Psi$

$$
\lim _{t \rightarrow \infty} H(u, t)=\Psi(u) .
$$

Hence $B(s, t) \rightarrow \phi(s)$ for Rl $s \geqq 0$ as $t \rightarrow \infty$. Also in (1.8) both sides are defined for Rl $s \geqq 0$ and are analytic for Rl $s>0$. Since both sides are equal for real $s$, it follows they are equal for all $s, \mathrm{Rl} s \geqq 0$.

Since $m(t) e^{-a t} \rightarrow c,(5.3),(5.4)$, and (5.6) imply that at all points of continuity of $\Psi$

$$
\lim _{t \rightarrow \infty} H_{1}(u, t)=\Psi(u) .
$$

In (5.5) this implies $B_{1}(s, t) \rightarrow \phi(s)$ as $t \rightarrow \infty, \mathrm{Rl} s \geqq 0$, which proves (1.6).

Addendum, November 3, 1958. The referee suggested weakening the requirement that $g(t)$ be continuous. By adding another stage to the proof, it can be shown that the results of this paper are valid for $G(t)$ absolutely continuous and $G(0+)=0$. This will now be done.

6. It will be assumed here that $G^{\prime}(t)=g(t) \geqq 0$ almost everywhere. Hence, there exists a sequence of continuous functions $\left\{g_{j}(t)\right\}$ such that

and

$$
g_{j}(t) \geqq 0, \quad \int_{0}^{\infty} g_{j}(t) d t=1,
$$

Let

$$
\lim _{j \rightarrow \infty} \int_{0}^{\infty}\left|g(t)-g_{j}(t)\right| d t=0 .
$$

$$
G_{j}(t)=\int_{0}^{t} g_{j}(y) d y
$$

By Theorem 2.1 there exist the generating functions $F_{j}(s, t)$, and

$$
F_{j}(s, t)=\int_{0}^{t} h\left(F_{j}(s, t-y)\right) g_{j}(y) d y+s\left[1-G_{j}(t)\right] ;
$$


that is, to each $G_{j}$ can be associated an $F_{j}$ satisfying the conclusion of Theorem 2.1 .

The argument used in Lemma 2.1 shows that if $k$ is chosen so that

$$
\mu \int_{0}^{\infty} e^{-k t} g(t) d t<\frac{1}{4}
$$

then by virtue of (6.1) it follows that if $j$ is large, then the inequality

$$
A(t) \leqq \mu \int_{0}^{t} A(t-y) g_{j}(y) d y+K
$$

implies that

$$
A(t) \leqq 2 K e^{k t}
$$

By using (6.2) for $j$ and $i$, where $i$ is also a positive integer,

$$
\begin{array}{r}
F_{j}(s, t)-F_{i}(s, t)=\int_{0}^{t}\left[h\left(F_{j}(s, t-y)\right)-h\left(F_{i}(s, t-y)\right)\right] g_{j}(y) d y \\
+\int_{0}^{t} h\left(F_{i}(s, t-y)\right)\left[g_{j}(y)-g_{i}(y)\right] d y+s\left[G_{i}(t)-G_{j}(t)\right] .
\end{array}
$$

Hence, since $\left|h^{\prime}\right| \leqq \mu$ and $|h| \leqq 1$,

$$
\begin{aligned}
\left|F_{j}(s, t)-F_{i}(s, t)\right| \leqq \mu \int_{0}^{t}\left|F_{j}(s, t-y)-F_{i}(s, t-y)\right| g_{j}(y) d y \\
+(1+|s|) \int_{0}^{t}\left|g_{j}(y)-g_{i}(y)\right| d y .
\end{aligned}
$$

Since the last term can be made as small as desired by taking $i$ and $j$ large enough, (6.3) implies that, for any $t_{0}, F_{j}(s, t)$ converges uniformly for $0 \leqq t \leqq t_{0},|s| \leqq 1$ as $j \rightarrow \infty$. If

$$
F(s, t)=\lim _{j \rightarrow \infty} F_{j}(s, t),
$$

then the uniform convergence guarantees that $F(s, t)$ is continuous for $|s| \leqq 1,0 \leqq t \leqq \infty$, and that $F(s, t)$ is a generating function for each $t$. Moreover, writing (6.2) as

$$
\begin{aligned}
F_{j}(s, t)=\int_{0}^{t} h\left(F_{j}(s, t-y)\right) g(y) d y & \\
& +\int_{0}^{t} h\left(F_{j}(s, t-y)\right)\left[g_{j}(y)-g(y)\right] d y+s\left[1-G_{j}(t)\right],
\end{aligned}
$$

it follows on letting $j \rightarrow \infty$, that $F(s, t)$ is a solution of (2.3).

Corresponding to each $g_{j}(t)$ there is, by Theorem 2.2 , an $m_{j}(t)$ satisfying

$$
m_{j}(t)=\mu \int_{0}^{t} m_{j}(t-y) g_{j}(y) d y+1-G_{j}(t) .
$$

Proceeding with the aid of $(6.3)$ much as before, it follows that $m_{j}(t)$ converges uniformly for $0 \leqq t \leqq t_{0}$, and hence

$$
m(t)=\lim _{j \rightarrow \infty} m_{j}(t)
$$


is a continuous nondecreasing function for $0 \leqq t \leqq \infty$ and satisfies (2.15). Also for large $j,(6.4)$ implies that

$$
m_{j}(t) \leqq 2 e^{k t} .
$$

From (6.5)

$$
\begin{gathered}
\frac{1-F_{i}\left(e^{-\sigma}, t\right)}{\sigma}-\frac{1-F_{j}\left(e^{-\sigma}, t\right)}{\sigma} \\
=\int_{0}^{t}\left[h\left(F_{j}\left(e^{-\sigma}, t-y\right)\right)-h\left(F_{i}\left(e^{-\sigma}, t-y\right)\right)\right] g_{j}(y) d y / \sigma \\
\quad+\int_{0}^{t} \frac{1-h\left(F_{i}\left(e^{-\sigma}, t-y\right)\right)}{1-F_{i}}\left[g_{i}(y)-g_{j}(y)\right] \frac{1-F_{i}}{\sigma} d y \\
+\frac{1-e^{-\sigma}}{\sigma} \int_{0}^{t}\left[g_{j}(y)-g_{i}(y)\right] d y .
\end{gathered}
$$

If we recall that by $(2.25) 1-F_{i}\left(e^{-\sigma}, t\right) \leqq \sigma m_{i}(t)$ and let

$$
\left|\left(1-F_{i}\right) / \sigma-\left(1-F_{j}\right) / \sigma\right|=W_{i j}(\sigma, t),
$$

the above yields

$$
\begin{aligned}
W_{i j}(\sigma, t) \leqq & \mu \int_{0}^{t} W_{i j}(\sigma, t-y) g_{j}(y) d y \\
& +\mu \int_{0}^{t}\left|g_{i}(y)-g_{j}(y)\right| m_{i}(t-y) d y+\int_{0}^{t}\left|g_{i}(y)-g_{j}(y)\right| d y
\end{aligned}
$$

By (6.6) for $0 \leqq t \leqq t_{0}$,

$$
\begin{aligned}
W_{i j}(\sigma, t) \leqq \mu \int_{0}^{t} W_{i j}(\sigma, t & -y) g_{j}(y) d y \\
& +\left(1+2 \mu e^{k t_{0}}\right) \int_{0}^{\infty}\left|g_{i}(y)-g_{j}(y)\right| d y .
\end{aligned}
$$

Since the last term can be made as small as desired by taking $i$ and $j$ large enough, (6.3) implies that $W_{i j}(\sigma, t)$ converges uniformly to zero for $0<\sigma<\infty$ and $0 \leqq t \leqq t_{0}$. Thus

$$
\left(1-F_{i}\left(e^{-\sigma}, t\right)\right) / \sigma-\left(1-F\left(e^{-\sigma}, t\right)\right) / \sigma
$$

converges uniformly to zero as $i \rightarrow \infty$ for $0<\sigma<\infty$ and $0 \leqq t \leqq t_{0}$, and hence as $i \rightarrow \infty$

$$
m_{i}(t)-\left(1-F_{i}\left(e^{-\sigma}, t\right)\right) / \sigma
$$

converges uniformly for $0<\sigma<\infty, 0 \leqq t \leqq t_{0}$, to

$$
m(t)-\left(1-F\left(e^{-\sigma}, t\right)\right) / \sigma .
$$

Since (6.7) is continuous and converges uniformly to zero as $\sigma \rightarrow 0+$, the 
uniform convergence of (6.7) to (6.8) implies that (6.8) is continuous on $0<\sigma<\infty, 0 \leqq t \leqq t_{0}$ and that

$$
\lim _{\sigma \rightarrow 0+}\left(1-F\left(e^{-\sigma}, t\right)\right) / \sigma=m(t),
$$

with the convergence uniform for $0 \leqq t \leqq t_{0}$, for any $t_{0}$.

From (2.28) onward, the argument now applies to the case $G(t)$ absolutely continuous by virtue of the above.

\section{REFERENCES}

1. Richard Bellman and Theodore Harris, On age-dependent binary branching processes, Ann. of Math. (2), vol. 55 (1952), pp. 280-295.

2. Norman Levinson, Limiting theorems for Galton-Watson branching process, Illinois J. Math., vol. 3 (1959), pp. 554-565.

Massachusetts Institute of Technology

Cambridge, Massachusetts 